\title{
Preparation and characterization of BSA and chitosan nanopartices for sustainable delivery system for quercetin
}

\author{
L. F. A. Anand Raj, R. Jonisha, B. Revathi, E. Jayalakshmy \\ Department of Biotechnology, St. Joseph's College of Engineering, Chennai, India.
}

\begin{tabular}{l}
\hline ARTICLE INFO \\
\hline Article history: \\
Received on: $13 / 03 / 2015$ \\
Revised on: $23 / 03 / 2015$ \\
Accepted on: $05 / 05 / 2015$ \\
Available online: $27 / 07 / 2015$ \\
\hline Key words: \\
BSA nanoparticle, Chitosan \\
nanoparticle, Quercetin, \\
sustainable release and SEM.
\end{tabular}

\begin{abstract}
The current study was undertaken in order to evaluate the efficacy of BSA and chitosan nanoparticle for sustainable delivery of Quercetin. BSA and chitosan nanoparticles were prepared by coacervation process and Ionic gelation method respectively and were characterized by Scanning Electron Microscope. The average size of BSA and chitosan nanoparticles was found to be $96 \mathrm{~nm}$ and $42.7 \mathrm{~nm}$ respectively. Quercetin loaded BSA and chitosan nanoparticles were smooth and spherical with the average size of $317.1 \mathrm{~nm}$ and $44.1 \mathrm{~nm}$ respectively. The encapsulation efficiency of the BSA and chitosan nanoparticles were calculated and it was found that the drug encapsulation efficiency was more in chitosan than in BSA. The in vitro analysis of Quercetin loaded BSA and chitosan nanoparticles were studied using dialysis bag. The nanoformulation of Quercetin loaded BSA nanoparticles shows a sustainable release of drug in which $56 \%$ of the drug was released till 24 hours and it did not change further. For Quercetin loaded chitosan nanoparticles it shows $72 \%$ of in vitro drug release for 24 hours and found to be better than BSA nanoparticle. Hence both Quercetin loaded BSA and chitosan nanoparticles were effective in encapsulation and sustainable release which also paves way for the bio availability of Quercetin
\end{abstract}

\section{INTRODUCTION}

Drug delivery is a promising approach for the biotechnologist and pharmacologist to develop a delivery system that can aid the drug to its target and maintaining its availability for their therapeutic action. Efficacy of most of the drugs were limited for several reasons such as poor absorption, poor solubility, nonspecific delivery, short circulating half life, high toxicity, high dosage etc (Orive et al., 2003; Praveen et al., 2012). It is always a dream for the scientist to develop a drug with the higher degree of specificity, solubility, improved efficacy with lesser side effects. Researchers have recognized the nanotechnology as a best solution to overcome the above said problem in developing an effective drug. Numerous nanotechnology platforms such as fullerenes, nanotubes, quantum dots, nanopores, dendrimers, liposomes, magnetic nanoprobes and radio controlled nanoparticles are being indentified so far (Faraji and Wipf, 2009). Nanoparticles that are compatible to the biological system have numerous applications

\footnotetext{
* Corresponding Author

L.F.A. Anand Raj, Department of Biotechnology, St. Joseph's College of Engineering, Chennai, India. E-mail: dranandlfa@gmail.com
}

in medicine (Chambers and Mitragotri, 2004; Surendran et al., 2009). They are considered as a best drug carrier system because of easy manipulation of particle size and surface, freedom to modify the fundamental properties such solubility, diffusivity and absorption (Jun et al., 2011). Moreover, smaller in size gives the unique properties of nanoparticle with greater surface area and distinct physical and chemical properties (Li et al., 2012) Drugs that are encapsulated in nanoparticles were found to provide enzymatic protection and sustained release that increases the bioavailability of the drug (Chambers and Mitragothri, 2004). It has been reported that nanoparticles can be a carrier for various pharmacological active substance such as phytochemicals, nutraceuticals and drugs (Rhaese et al., 2003; Zhang et al., 2008). Among numerous nanoparticle, Bovine Serum Albumin (BSA) and chitosan based drug carrier are currently in research in order to deliver various drugs such as low molecular drug, anticancer drug, DNA, vaccine etc (Taheri et al., 2012). BSA nanoparticles are non toxic, biodegradable and biocompatible material, which are easily adaptable to the human body (Jahanshahi et al., 2008; Jun et al., 2011). It has potential usefulness in transporting fatty acids, endogenous and exogenous compounds throughout the body (Fang et al., 2011; Ascenzi et al., 2006; Ascoli et al., 2006). 
BSA are rich in charged amino acids (lysine) that allow the positive and negative charged molecule to adsorb electro statically without the participation of any other compounds (Tan et al.,2013; Rabea et al., 2003; Jayakumar et al., 2010; Patel et al., 2012; Namasivayam et al., 2014). Chitosan is a polycationic polysaccharide distributed in crustacean kingdom. It is used for biomedical application such as scaffold in tissue engineering, wound dressing, drug carrier and antimicrobial agent (Jameela and Jayakrishnan, 1995; Scheffel et al., 1973; Sahoo et al., 2011). Chitosan nanoparticle is unique in targeting the drugs to various organs such as liver, colon, kidney and lungs and thus provides a suitable carrier for various drugs especially for cancer (Chakraborty et al., 2012). Quercetin (3, 5, 7, 3, 4' pentahydroxyflavone) is bioflavonoid found in some fruits vegetables, food and beverages (Formica and Regelson, 1995). It forms the backbone for other flavonoids such as rutin, hesperidins, Naringenin and tangeritin (Lakhanpal and Rai, 2007). It is an antioxidant and found to regulate lipid peroxidation by chelating metal ions and also used as a radioprotector against UV radiation induced injury and cell death (Kandaswami et al.,2005). It is a potent pharmacological active agent which has a wound healing, anti-inflammatory, anticancerous and antiviral properties (Comalada et al., 2005; Kakran and Sahoo, 2011; Jagtap et al., 2009). Quercetin incorporated collagen matrices were found to be a potent wound healer (Gomathi et al., 2003). In spite of its pharmacological properties, its use is highly limited because of poor solubility and poor absorption in oral administration (Kakran and Sahoo, 2011). Hence the formulation of Quercetin that enhances the bioavailability will improve its pharmacological action. It has been reported that nanovehicle can overcome the above said problem. In this aspect our purpose of the current study is to evaluate the production and characterization of BSA and chitosan nanoparticle and to study the efficacy of Quercetin encapsulation and sustainable release of Quercetin from BSA and chitosan nanoparticle

\section{MATERIALS AND METHODS}

The chemicals for the preparation of BSA and chitosan nanoparticles were obtained from Reachem Chemicals. Quercetin (Extra pure) was obtained from Sisco Laboratories, Mumbai. All other chemicals used were analytical grade and high purity chemicals were purchased from SRL chemicals. Deionized water was used throughout the experiment.

\section{Preparation of BSA Nanoparticles}

The method adopted for the preparation of BSA nanoparticle was desolvation method (Jahanshahi et al., 2008; Langer et al., 2003). 1\% BSA was prepared in deionised water at $\mathrm{pH} 7$ and titrated with acetone by stirring at $700 \mathrm{rpm}$ using magnetic stirrer at room temperature until the solution turned turbid (Sailaja and Amareshwar, 2012). 25\% of Glutaraldehyde was added drop wise as a cross linker and the mixture was maintained in stirring condition for 3 hours. The resulting mixture was centrifuged at 5000rpm for 30 minutes. The supernatant was discarded. The pellet was washed with acetone and then with deionized water for further purification. The pellet was collected, dried and characterized.

\section{Preparation of Chitosan Nanoparticles}

Ionic gelation method was followed for the preparation of chitosan nanoparticles (Calvo et al., 1997). 0.3\% chitosan was prepared in $1.5 \%$ of acetic acid. The chitosan nanoparticles were prepared with drop wise addition of Sodium Tripolyphosphate $(1 \mathrm{mg} / \mathrm{ml})$ under magnetic stirring at room temperature for $1 \mathrm{hr}$. The resulting mixture was centrifuged at $15000 \mathrm{rpm}$ for 20 minutes. The pellet was collected and was used for characterization.

\section{Quercetin loaded in BSA Nanoparticles}

The BSA nanoparticles were loaded with the drug Quercetin and were checked for its entrapment efficiency. The protocol for the encapsulation of the drug was referred from the studies led by Namasivayam et al., (2014) with minor modification. 1\% BSA was prepared and $0.5 \mathrm{~g}$ of Quercetin was dissolved in it. The solution was titrated with acetone until the solution became turbid. $25 \%$ of glutaraldehyde was added to the solution for cross linking and the solution was agitated for 3 hours in a magnetic stirrer. The solution was ultrasonicated to reduce the size of the particle which was then centrifuged at 5000rpm for 30 minutes. The supernatant was subjected for encapsulation efficacy and the pellet was collected for further characterization.

\section{Quercetin loaded in Chitosan Nanoparticles}

$0.15 \mathrm{~g}$ of Quercetin was dissolved in $0.3 \%$ of chitosan prepared in $1.5 \%$ of acetic acid which was titrated against Sodium Tripolyphosphate (TPP) kept in magnetic stirrer. The solution was then subjected to ultrasonication which reduced the particle size and centrifuged at 5000rpm for 30 minutes. The supernatant was subjected for encapsulation efficacy and the pellet was collected for further characterization.

\section{Encapsulation Efficiency}

To check the encapsulation efficiency, the free Quercetin that was present in the supernatant after centrifugation was measured in UV Spectrophotometer at $370 \mathrm{~nm}$. The encapsulation efficiency was calculated using the formula:

Entrapment Efficiency $(\%)=$

$\frac{\text { Total Quercetin - Quercetin in supernatant } * 100}{\text { Total Quercetin }}$

\section{In vitro drug release}

In order to study the in vitro drug release dialysis bag from Himedia (Membrane -50) was used. $10 \mathrm{mg}$ of the Quercetin loaded BSA and Chitosan nanoparticles were taken and were dispersed in $1 \mathrm{ml}$ of phosphate buffer $(0.1 \mathrm{M} / \mathrm{L})$ solution which was placed in a Himedia dialysis membrane bag. The dialysis membrane bag was immersed in $150 \mathrm{ml}$ phosphate buffer solution 
which was continuously stirred. $5 \mathrm{ml}$ of samples were collected at definite time intervals which were replaced with phosphate buffer solution. The drug release was determined by taking O.D in UVVis Spectrophotometer at 370nm (Namasivayam et al., 2014; Shaikh et al., 2009).

\section{Characterization of BSA and Chitosan nanoparticles}

The produced BSA and Chitosan nanoparticles were characterized using Scanning Electron Microscope (SEM) analysis, Carl Zeiss, Germany before and after encapsulation the drug reveals the size, shape and structure of the nanoparticle. It also tells whether the drug has been loaded into the prepared BSA and chitosan nanoparticles.

\section{RESULTS AND DISCUSSION}

\section{Characterization of BSA and Chitosan Nanoparticle}

The size and shape of nanoparticle was determined by using Scanning Electron Microscope. It was clear that most of the morphologies of both chitosan and BSA nanoparticle were semisphere with diameter ranges between $25-120 \mathrm{~nm}$. The images of the nanoparticle for BSA and chitosan were illustrated in Fig 1 and Fig 2 respectively. The average size of BSA nanoparticle was $96 \mathrm{~nm}$ which matched with previous study.

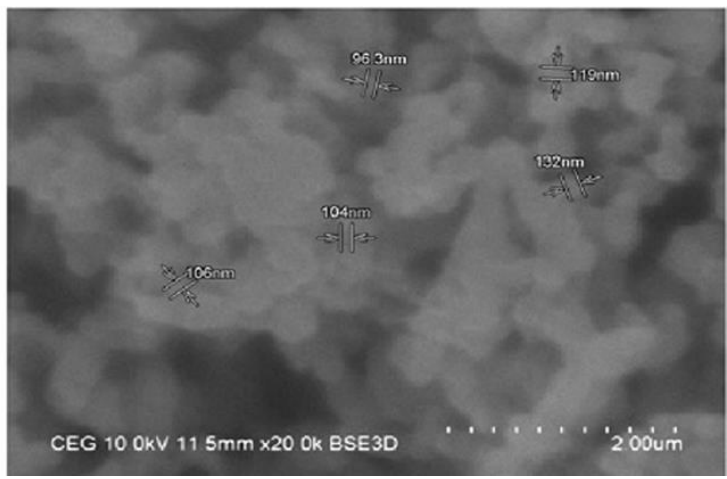

Fig. 1: SEM image of BSA nanoparticle.

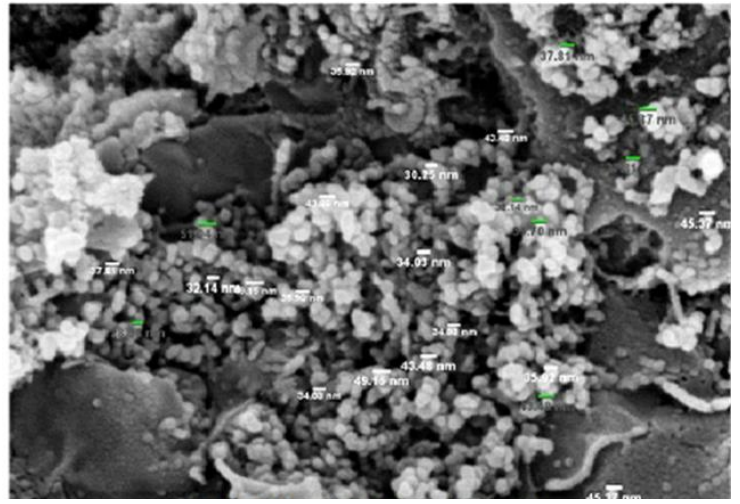

Fig. 2: SEM image of chitosan nanoparticle.

According to Jahanshahi et al, (2008) the average size of the BSA nanoparticle was influenced by the concentration and it was found that the greater concentration had a lesser particle average size of $101 \mathrm{~nm}$. The size of the nanoparticles depends on the ration of chitosan and TPP. TPP is a positive charge ions that interacts with the negatively change amino group of chitosan to form a nanoparticles. The size of the chitosan nanoparticle showed a minimum size of $28.3 \mathrm{~nm}$ and maximum of $46 \mathrm{~nm}$. Preparation of chitosan nanoparticle by ionic gelation was widely used. This technique offers a simple and rapid production with minimum nanoparticle size. Earlier reports suggest the size and surface of chitosan nanoparticle can be modified by varying the ratios of chitosan and stabilizer (Calvo et al., 1997).

\section{Encapsulation Efficiency of BSA and Chitosan Nanoparticle}

On encapsulating the Quercetin with BSA nanoparticles the size of the particle were found to increase. Earlier reports suggested that the encapsulation efficiency of BSA nanoparticle was due to the electrostatic and hydrophobic interaction between the drug and nanoparticle and this could be a reason for the increase in size of the particle (Yu et al, 2014).

Fig. 3 represents the SEM image of the BSA nanoparticle that showed some structural changes when loaded with Quercetin. On the other hand, when chitosan was loaded with Quercetin (Fig. 4), no significant increase in size was observed.

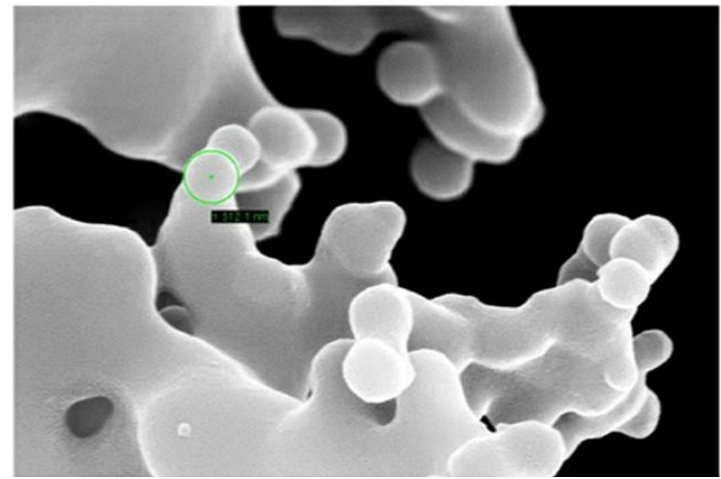

Fig. 3: SEM image of quercetin loaded BSA nanoparticles.

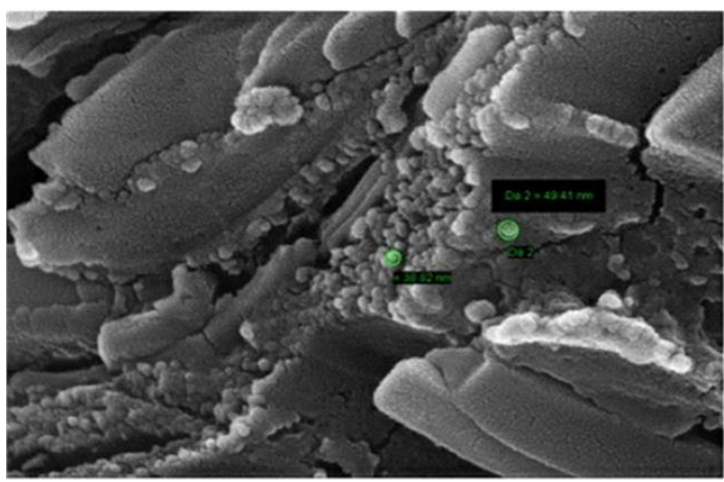

Fig.4: SEM image of quercetin loaded chitosan nanoparticles.

The encapsulation efficiency of the BSA and chitosan nanoparticles were calculated and it was found that the drug encapsulation efficiency was more in chitosan than in BSA. According to $\mathrm{Wu}$ et al (2005) chitosan a low molecular weight polysaccharide provides stability to the drug in suspension. The drug encapsulation efficiency of BSA and chitosan nanoparticle loaded with Quercetin is shown in Table.1 
Table 1: Drug encapsulation efficiency of BSA and Chitosan nanoparticles.

\begin{tabular}{lcc}
\hline S.No & Nanoparticle & \% of encapsulation \\
\hline 1 & BSA & 59 \\
2 & Chitosan & 61 \\
\hline
\end{tabular}

\section{In vitro drug release of BSA and chitosan nanoparticle}

The in vitro drug release was studied in BSA and chitosan nanoparticles using the dialysis method. Fig 5 displayed a graph showing the in vitro drug release of BSA nanoparticles in which $56 \%$ of the drug was released till 24 hours and was not found to change further. Fig 6 displayed a graph showing $72 \%$ of in vitro drug release of chitosan nanoparticles. The in vitro drug release profile of BSA was studied by $\mathrm{Yu}$ et al (2014) which confirmed that BSA nanoparticle exhibited a good sustainable release profile due to which it can be used a drug delivery system. Similarly Dounighi et al (2012) studied the in vitro release of scropian venom as an antigen delivery system in which the chitosan nanoparticle showed an initial burst of release of approximately $60 \%$ in first ten hours, which was in accordance with our results.

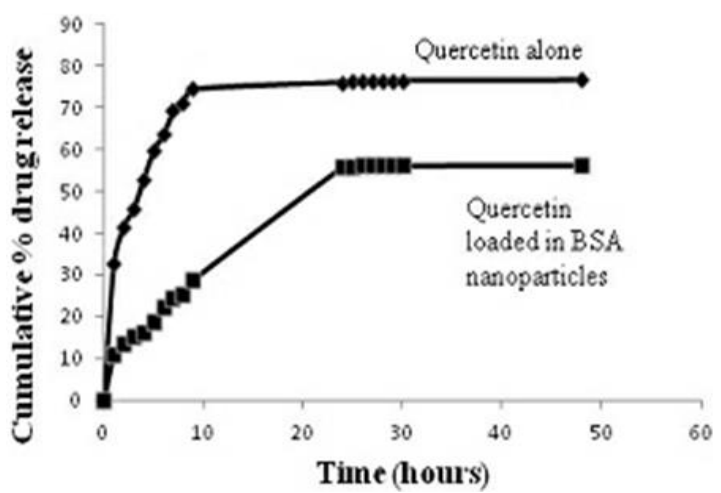

Fig. 5: Graph showing cumulative drug release of quercetin loaded BSA nanoparticles.

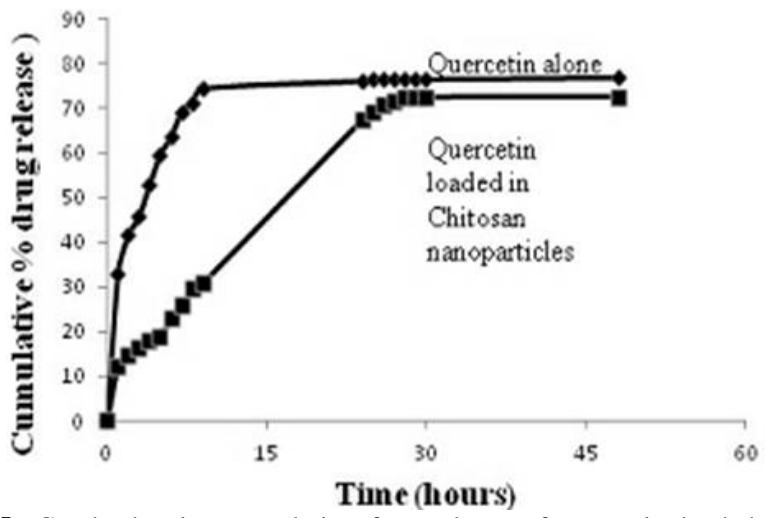

Fig. 5: Graph showing cumulative frug release of quercetin loaded BSA nonparticles.

\section{CONCLUSION}

In this study, BSA and chitosan nanoparticles were prepared by using desolvation and ionic gelation method and this was confirmed by SEM analysis with the minimum particle size. These nanoparticles also showed a maximum Quercetin encapsulation efficiency. It was clear that successful encapsulation of Quercetin in chitosan nanoparticle will provide the bioavailability of Quercetin in the system. The pharmacological properties of Quercetin and their uses in medicine were limited due to their poor availability our encapsulation result for BSA and chitosan nanoparticle overcomes their Quercetin limitation by increasing their availability and thus BSA and chitosan can be considered as an attractive polymers that can be used as a good carrier for drugs. The in vitro studies reveal that the BSA and chitosan nanoparticle loaded with Quercetin showed a sustainable release of about $56 \%$ and $72 \%$ respectively. Therefore the current study suggest that the pharmacological properties of Quercetin can be increased by using both BSA and chitosan nanoparticle as a good drug delivery system. From the present study it was found that chitosan nanoparticle when compared with BSA showed increased encapsulation efficiency and sustainable release of the Quercetin. Further studies have to be done by encapsulating other drugs into the chitosan nanoparticle to make it as a better drug delivery system.

\section{ACKNOWLEDGEMENT}

The authors are thankful for the Department, Staff and Management of St .Joseph's College of Engineering, Chennai.

\section{REFERENCES}

Ascenzi P, Bocedi A, Notari S, Fanali G, Fesce R, Fasano M: Allosteric modulation of drug binding to human serum albumin. Mini Rev Med Chem, 2006; 6:483-489.

Ascoli G, Domenici E, Bertucci C: Drug binding to human serum albumin: Abridged review of results obtained with highperformance liquid chromatography and circular dichroism. Chirality, 2006; 18:667-679.

Calvo P, Remunan-Lopez C, Vila-Jato JL, Alonso MJ. Chitosan and chitosan /ethylene oxide propylene oxide block copolymer nanoparticles as novel carriers for proteins and vaccines. Pharm. Res., 1997; 14:1431-1436.

Chakraborty S, Stalin S, Das N, Choudhury ST, Ghosh S, Snehasikta S. The use of nano-Quercetin to arrest mitochondrial damage and MMP-9 upregulation during prevention of gastric inflammation induced by ethanol in rat. Biomaterials, 2012; 33:29913001.

Chambers E and Mitragotri S. Prolonged circulation of large polymeric nanoparticles by non-covalent adsorption on erythrocytes. J Controlled Release, 2004; 100:111- 119.

Comalada M, Camuesco D, Sierra S, Ballester I, Xaus J, Galvez J, Zarzuelo A. In vivo quercitrin anti-inflammatory effect involves release of Quercetin, which inhibits inflammation through downregulation of the NF kappa B pathway. Eur J Immunol, 2005; 35:584-592.

Dounighi M N, Eskandari R , Avadi MR, Zolfagharian H , Sadeghi MMA and Rezayat M. Preparation and in vitro characterization of chitosan nanoparticles containing Mesobuthus eupeus scorpion venom as an antigen delivery system. The Journal of Venomous Animals and Toxins Including Their Tropical diseases, 2012; 18:44-52.

Fang R, Jing H, Chai Z, Zhao G, Stoll S, Ren F, Liu F, Leng X. Design and characterization of protein-Quercetin bioactive nanoparticles. J. Nanobiotechnology, 2011; 9:1-14.

Faraji AH and Wipf P. Nanoparticles in cellular drug delivery. Bioorg Med Chem, 2009; 17:2950-2962.

Formica JV and Regelson W. Review of the biology of Quercetin and related bioflavonoids. Food Chem Toxicol, 1995; 33:10611080 . 
Gomathi, K. Gopinath, D. Ahmed, M. R. Jayakumar, R. Quercetin incorporated collagen matrices for dermal wound healing processes in rat. Biomaterials, 2003; 24:2767-2772.

Jagtap S, Meganathan K, Wagh V, Winkler J, Hescheler J and Sachinidis A. Chemoprotective mechanism of the natural compounds, epigallocatechin-3-O-gallate, Quercetin and curcumin against cancer and cardiovascular diseases. Med Chem, 2009; 16: 1451-1462.

Jahanshahi M, Najafpour G, Rahimnejad M. Applying the Taguchi method for optimized fabrication of bovine serum albumin (BSA) nanoparticles as drug delivery vehicles. Afr. J. Biotechnol, 2008; 7:362367.

Jameela SR and Jayakrishnan A. Glutaraldehyde cross-linked chitosan microspheres as a long acting biodegradable drug delivery vehicle: studies on the in vitro release of mitoxantrone and in vivo degradation of microspheres in rat muscle. Biomaterials, 1995; 16:769-75.

Jayakumar R, Prabaharan M, Nair SV, Tamura H. Novel chitin and chitosan nanofibers in biomedical applications. Biotechnol. Adv, $2010 ; 28: 142-150$

Jun J, Nguyen HH, Paik SYR, Chun HS, Kang BC, Ko S. Preparation of size-controlled bovine serum albumin (BSA) nanoparticles by a modified desolvation method. Food Chem, 2011; 127:1892-1898.

Kakran M, Sahoo NG, Li L. Dissolution enhancement of Quercetin through nanofabrication, complexation, and solid dispersion. Colloids and Surfaces B: Biointerfaces, 2011; 88:121- 130.

Kandaswami C, Lee LT, Lee PP, Hwang JJ, Ke FC, Huang YT, Lee MT. The antitumor activities of flavonoids. In Vivo, 2005; 19:895909.

Lakhanpal P, Rai DK. Quercetin: A Versatile Flavonoid. Internet Journal of Medical Update, 2007; 2:22-37.

Langer K, Balthasar S, Vogel V, Dinauer N, Von Briesen H, Schubert D. Optimization of the preparation process for human serum albumin (HSA) nanoparticles. Int. J. Pharm., 2003; 257(1-2):169-180.

Li X, Chen M, Yang W, Zhou Z. Interaction of bovine serum albumin with self-assembled nanoparticles of 6-O-cholesterol modified chitosan. Colloids and Surfaces B: Biointerfaces, 2012; 92:136-141.

Namasivayam SKR, Raj LFAA, Robin AT, Chandra JH, Bharani RSA. Optimal Synthesis of Biocompatible Bovine Serum Nanoparticles-Incorporated Quercetin (BSA NPS-QT) Nano Drug Conjugate for the Controlled Release and Improved Anti Oxidative Activity. Res. J. Pharm., Biol., Chem. Sci, 2014; 5:478-487.

Orive G, Hernandez RM, Rodriguez Gascon A, Dominguez-Gil A, Pedraz JL. Drug delivery in biotechnology: present and future. Curr Opin Biotechnol 2003; 14:659-64.

Parveen S, Misra R, Sanjeeb KS. Nanoparticles: a boon to drug delivery, therapeutics, diagnostics and imaging. Nanomedicine: Nanotechnology, Biology, and Medicine, 2012; 8:147-166.

Patel K, Shrimanker M, Dave R, Modi H, Anand J Bhadani S. Preparation and In Vivo Study Of Doxorubicin Hcl Loaded Chitosan Nanoparticles Prepared By W/O Emulsion Method. International Journal of Current Research, 2012; 4:438-440.
Rabea EI, Badawy ME, Stevens CV, Smagghe G, Steurbaut W. Chitosan as antimicrobial agent: Applications and mode of action. Biomacromolecules, 2003; 4:1457-1465.

Rhaese S, Briesen VH, Waigmann RH, Kreuter J, Langer K. Human serum albumin-polyethyleneimine nanoparticles for gene delivery. J Controlled Release, 2003; 92:199-208.

Sailaja AK and Amareshwar. P. Preperation of BSA nanoparticles by desolvation technique using acetone as a desolvating agent. International Journal of Pharmaceutical Science and Nanotechnology, 2012; 5:1643-1647.

Sahoo NG, Kakran M, Shaal LA, Li L, Mu“ Ller RH, M. Pal, Tan LP. Preparation and Characterization of Quercetin Nanocrystals. J. Pharm. Sci, 2011; 100:2379-2390.

Scheffel, U, Rhodes, BA, Natarajan TK, Wagner Jr HN. Albumin microspheres for study of the reticuloendothelial system. J. Nucl. Med, 1973; 13:498-503.

Shaikh J, Ankola D D, Beniwal V, Singh D, Kumar MN Nanoparticle encapsulation improves oral bioavailability of curcumin by atleast 9-fold when compared to curcumin administered with piperine as absorption enhancer. Eur. J. Pharm. Sci, 2009; 37:223-230.

Surendiran A, Sandhiya S, Pradhan SC ,Adithan C. Novel applications of nanotechnology in medicine. Indian J Med Res, 2009; 130:689-701.

Taheri ES, Jahanshahi M, Taghi M, Mosavian H. Preparation, Characterization and Optimization of Egg Albumin Nanoparticles as LowMolecular-Weight Drug DeliveryVehicle. Part. Part. Syst. Charact, 2012; 29:211-222

Tan H, Lin RMC, Liu Z Tang T. Quaternized Chitosan as an Antimicrobial Agent: Antimicrobial Activity, Mechanism of Action and Biomedical Applications in Orthopedics. Int J mol sci, 2013; 14:18541869.

Wu Y, Yang W, Wang C, Hu J, Fu S. Chitosan nanoparticles as a novel delivery system for ammonium glycyrrhizinate. Int.J.Phar, 2005; 235-245.

Yu Z, Yu M, Zhang Z, Hong G and Xiong Q. Bovine serum albumin nanoparticles as controlled release carrier for local drug delivery to the inner ear. Nanoscale Res. Lett, 2014; 9:1-7.

Zhang L, Gu FX, Chan JM, Wang AZ, Langer RS, Farokhzad OC. Nanoparticles in Medicine: Therapeutic Applications and Developments. Clin Pharmacol Ther, 2008; 83:761-769.

\section{How to cite this article:}

Anand Raj L.F.A., Jonisha R., Revathi B., Jayalakshmy E. Preparation and Characterization of bsa and chitosan Nanopartices for Sustainable delivery system for Quercetin. J App Pharm Sci, 2015; 5 (07): 001-005. 